\title{
Targeted Therapy of Breast Cancer - New Insights and Perspectives
}

\author{
Joachim Bischoff \\ Universitätsfrauenklinik Magdeburg, Germany
}

\section{Receptor-Based Therapy}

Current targeted therapy of breast cancer is strongly associated with the term receptor tyrosine kinase. All agents of this new world of antineoplastic therapy that have been approved so far are directed against members of this receptor superfamily. The clinical significance of targeted therapy is defined by synergistic effects with conventional chemotherapy. The HER2-targeted therapy shows that inhibition of a single receptor can be highly efficient in the treatment of breast cancer. However, limitations of the first generation of targeted treatment are apparent by the example of the HER 2 antibody trastuzumab.

\section{What have we Learnt from Trastuzumab - Overcoming Resistance}

A significant number of patients with HER2-positive breast cancer do not respond to therapy with trastuzumab. Others who initially respond to the agent develop a secondary resistance in the further course of treatment. Several mechanisms responsible for this development have been identified. Based on these findings, the establishment of further anti-HER2 therapies is expected during the upcoming years.

\section{Novel HER2-Directed Antibodies}

Current promising candidates are trastuzumab-DM1 (T-DM1) [1], an antibody drug conjugate (ADC), or pertuzumab [2], that inhibit dimerisation of the HER2 receptor by binding to the corresponding epitope in the extracellular domain. Upon binding of the ADC surface tumour antigens (i.e. HER2), the ADC-receptor complex is internalised into the cell where the cytotoxic drug is released. This investigational ADC has a proposed dual mechanism of action: anti-HER2 activity and targeted intracellular delivery of DM1, a maytansine derivative that is a potent antimicrotubule agent. T-DM1 has shown activity in trastuzumab-pretreated patients as a single agent. Pertuzumab has been investigated in combination with trastuzumab also in HER2-positive breast cancer with encouraging results.

Furthermore, it may be possible to enhance the efficacy of antibodies by modification of the sugar compound. There is evidence that modifications in the sugar compound will enhance the efficacy of antibodies. Removal of fucose significantly increases the strength of the bond between antibody and the immunocompetent cell, which leads to enhanced antibody-dependent cell-mediated cytotoxicity. Clinical trials investigating two of these defucosylated antibodies are currently under way.

\section{Small Molecules}

An alternative approach for optimising HER2-targeted therapy is the implementation of tyrosine kinase inhibitors. Studies conducted with lapatinib, a dual tyrosine kinase inhibitor of HER1/2, demonstrated the significance of this drug class in the treatment of breast cancer. Lapatinib in combination with capecitabine was the first targeted agent shown to be efficient after pre-treatment with trastuzumab [3].

An advance of this therapeutic principle leads to the complete inhibition of tyrosine kinase activity in all members of the HER family with an active catalytic site (HER1/2/4) by panHER inhibitors. In contrast to lapatinib, these pan-HER inhibitors bind irreversibly to the adenosine triphosphate (ATP) binding pocket of the intracellular receptor domain, which might result in improved efficacy. First agents of this new generation of small molecule inhibitors such as neratinib (TKI

\section{KARGER}

Fax +497614520714

Information@Karger.de

www.karger.com (c) 2008 S. Karger GmbH, Freiburg

Accessible online at:

www.karger.com/brc

Universitätsfrauenklinik Magdeburg A.ö.R.

Gerhart-Hauptmann-Straße 35, 39108 Magdeburg, Germany

Tel. +391 671731-0, Fax -7

joachim.bischoff@med.ovgu.de 
272) are currently under clinical investigation. A phase II open label study with 102 patients with advanced metastatic breast cancer (MBC) was recently reported. Patients not experiencing prior standard treatment for breast cancer with trastuzumab had a progression-free survival (PFS) rate of $75 \%$ while patients with prior trastuzumab treatment had a 16-week PFS of $51 \%$ [4].

\section{Antiangiogenesis}

To date, antiangiogenic therapy is based on the inactivation of the vascular endothelial growth factor receptor (VEGFR) by antibody-mediated antagonism of the ligand VEGF. Bevacizumab has been approved for the first-line therapy of MBC in combination with paclitaxel but has failed to show a survival benefit. According to preclinical models, it is speculated that angiogenesis is essential in the early period of carcinogenesis. Therefore, prospective trials evaluating bevacizumab in the adjuvant and neoadjuvant setting have been initiated. On the other hand, tumour angiogenesis is a multistep process involving multiple growth factor receptors, such as platelet-derived growth factor receptor (PDGFR) and FMS-like tyrosine kinase 3 (FLT3).

\section{Multi Targeting}

These considerations provide the rationale for conducting future studies focusing on so-called multikinase inhibitors that bind to several intracellular domains of tyrosine kinase receptors concurrently. Preliminary results indicate that these multikinase inhibitors are also active in breast cancer as recently published for sunitinib. The substance targets several receptor tyrosine kinases, including VEGFR (VEGFR-1, VEGFR-2, VEGFR-3), PDGFR (PDGFR- $\alpha$, PDGFR- $\beta$ ), KIT, and colony-stimulating factor-1 receptor [5]. Based on the findings of a phase I study evaluating the feasibility of sunitinib plus docetaxel, a phase III trial was conducted comparing this combination with the taxane monotherapy. Sunitinib is the first multikinase inhibitor to become subject of a large-scale study program in breast cancer.

\section{Downstream Signalling}

Signalling transduction is triggered by a broad spectrum of second messenger molecules that additionally feature an increasing number of genetic alterations in the course of tumorigenesis. This might be a cause for limited success of receptorbased therapies in advanced stages of disease. In this context, the serine/threonine kinase mammalian target of rapamycin (mTOR) is of special concern. mTOR is part of the PI3K/Akt pathway. Constitutive PI3K/Akt activity was previously shown to inhibit cell cycle arrest and apoptosis mediated by trastuzumab. The clinical relevance of mTOR inhibition by rapamycin analogues such as temsirolimus and everolimus (RAD001) was already confirmed in other indications, e.g. renal cell carcinoma [6]. With its function as downstream mediator within the HER2 pathways, mTOR might also be considered as a potential target in breast cancer. A matter of particular interest is therefore whether mTOR inhibitors are able to break through trastuzumab resistance, mediated by pTEN deficiency. Decreased levels of the pTEN phosphorylation and signalling reverse trastuzumab-mediated growth arrest of HER2-overexpressing breast cancer cells. Another important signalling cascade downstream of growth factor receptors is the Ras/Raf/MEK/ERK pathway.

\section{Receptor Cross Talk}

The activity of growth factor receptors is regulated within complex signalling networks. In breast cancer, especially the cross talk between HER2 and VEGFR has been well investigated [7]. Emerging clinical data indicate that efficient therapeutic strategies can be derived from receptor cross talk. A combination of trastuzumab and bevacizumab was the first regimen without chemotherapy to reach a response rate of about $50 \%$ as first-line treatment in HER2-positive tumours [8]. A randomised phase III trial comparing lapatinib versus a combination of lapatinib and pazopanib, a VEGFR tyrosine kinase inhibitor, recently confirmed the feasibility of a dual HER2-VEGFR inhibition [9].

Interactions between HER2 and insulin-like growth factor 1 receptor (IGF1R) are well documented. IGF1R overexpression appears to be associated with the loss of trastuzumab efficiency. Inhibitory effects of lapatinib on the IGF1R pathway have been demonstrated in trastuzumab-resistant breast cancer cell lines. This effect could be enhanced by combining lapatinib with an IFG1R antibody [10].

The influence of tyrosine kinase receptors on mechanisms of endocrine resistance might open new dimensions in treating the hormone receptor-positive breast cancer. Oestrogen receptor (ER) functions as a ligand-dependent transcription factor. $\mathrm{ER}$, in its classical action, directly binds to DNA sequences called oestrogen response elements (EREs) residing in the promoter region of target genes, and by recruiting coregulatory proteins that regulate gene transcription. Importantly, signalling from different growth factor receptor-dependent kinases phosphorylates various factors in the ER pathway, including ER itself; this potentiates ER genomic signalling activity on gene transcription. However, in the presence of hyperactive growth factor receptor signalling, as often occurs in breast cancer (e.g. HER2 overexpression), an excessive phosphorylation of ER and its coregulators may severely weaken the inhibitor effects of various endocrine therapies and lead to endocrine resistance. The combination of ER-tar- 
geted therapies with growth factor receptor inhibitors or inhibitors of more downstream kinases is a novel strategy currently undergoing more intensive clinical investigation. Interestingly, resistance to lapatinib was recently shown to be mediated by increased signalling from the ER in ER-positive HER2-overexpressing breast cancers, suggesting that co-targeting of ER and HER2 may be beneficial in this population $[11,12]$. A proof-of-concept study demonstrated a certain amount of activity for the combination of anastrozol, an aromatase inhibitor, and trastuzumab in HER2-positive MBC [13].

\section{Further Combinations of Targeted Agents}

Interactions between respective therapeutic targets within single pathways also provide the rationale for examining synergistic effects between targeted drugs. Due to the increasing number of activated oncogenes in the course of disease, there is an unmet need for such a kind of multi-targeted drug combination on the way to more effective anti-cancer treatment. Dual HER2 blockade with lapatinib plus trastuzumab represents the first combination therapy with antibodies and small molecules that has proven superior activity in MBC compared to lapatinib alone. Results of disease control (clinical benefit rate (CBR) $25 \%$, overall survival (OS) 52 vs. 39 weeks) are highly clinically relevant because patients received a median of 3 trastuzumab-containing regimens [14].

\section{Sequential Regiments (Treatment in Multiple Lines)}

The number of therapeutic options based on cytotoxic drugs as well as treatment duration was generally limited by increasing chemoresistance and the appearance of cumulating toxicities in the course of disease. The taxan-trastuzumab combination as first-line therapy in HER2-positive breast cancer leads to a significant increase in overall survival, providing a demonstration of the general potential of targeted agents $[15,16]$. However, a curative chance is missing in MBC even under biologicals, due to the development of secondary or primary resistance. The intermediate aim is to reach a better outcome in patients with palliative therapy by sequentially combining non-cross-resistant therapy regimens. Due to selective targeting in the signalling network, the absence of cumulative toxicities and a favourable profile of side effects, biologicals are suitable agents for sequential use. Further progress can be expected in the near future, by integration of the most promising agents into standard protocols to prolong time to progression, as shown by administration lapatinib plus capecitabine after trastuzumab pre-treatment. Prospective studies are required to find out if sequential administrations of biologicals can induce long-term disease control and turn metastatic breast cancer successively into a chronic disease.

\section{Maintenance Therapy}

Metastatic breast cancer is usually treated with the same regimen until progression. However, feasibility is impaired by cumulative toxicities, particularly with combination therapy. The introduction of biologicals offers new perspectives. For example, after achieving remission with trastuzumab-based combination therapy, it has been efficient to maintain therapy with the HER2 antibody. This approach was associated with longterm stable disease in several cases. Moreover, maintenance therapy with angiogenesis inhibitors subsequent to combination therapy with cytotoxics and angiogenesis inhibitors broadens the means to optimise treatment. This concept needs further evolution within randomised controlled trials.

\section{Targeting Breast Cancer Stem Cells}

Recent evidence has shown that resistance against conventional systemic therapy is associated with breast cancer stem cells. They are characterised by the ability of self-renewal [17]. Dysregulation of this process results in the clonal expansion of stem cells, which marks a central step in carcinogenesis. Activation of the hedgehog, notch, and Wnt- $\beta$-catenin signal transduction pathways $[8,16,18]$ is essential in this process. However, presumably none of these had relevant impact on breast cancer in the past. Deactivation of known suppressor genes like BRCA1 in hereditary and PTEN in sporadic breast cancer is of special significance in this context. In addition, HER2 expression has been identified as stem cell marker [19]. A neoadjuvant study revealed that lapatinib, the dual HER1/2 inhibitor, reduced the population of stem cells, and induced increased pathologic complete remission. In contrast, neoadjuvant chemotherapy induces a stem cell increase [20]. The drug design of new agents, targeted against mediators of stem cellassociated pathways, will open a gateway to new therapeutic options. Inhibitors of the hedgehog [21] signal transduction pathway as well as antagonists of $\chi$-secretase [22], an enzyme of the notch signalling pathway, are currently subjects of clinical studies.

\section{Predictive Factors}

The growing number of targeted agents provides the opportunity for tailoring treatment according to the needs of an individual patient, provided the respective patients can be identified by the targets. Experience has shown that problems may arise. So far, the ER and the HER2 receptor have been successfully established as cellular targets for endocrine and trastuzumab therapy, respectively, making them predictive factors for treatment response. Molecular markers of downstream signalling also function as predictive factors for receptor-based therapies, as recently documented for the K-ras mu- 
tation in colon cancer resistant to anti-EGFR antibody treatment, while the wild type shows a high response. The K-ras test was introduced into clinical routine later than the EGFR antibodies cetuximab and panitumumab, which mirrors the fast development in the field of targeted therapies and their possible consequences in oncology.

\section{Evaluation of Response}

Considering the mode of action of these new non-cytotoxic drugs, their mono-activity measured as attainable remission rate is small in the majority of cases. Instead, they are marked by their synergistic interaction with cytotoxic drugs. It has yet to be examined whether radiological methods like computed tomography and magnetic resonance imaging can display the efficacy of these biologicals. In the case of imatinib, a first-generation kinase-inhibitor in GISTumours, an early response was detectable within a few days by positron emission tomography. Further examinations are necessary to find out if molecu- lar imaging can adequately display progress in tumour staging for other targeted drugs.

\section{Conclusion}

In light of the number of targeted agents that are currently being developed, identifying those breast cancer patients who will profit from tailored therapy will remain a challenge. So far, only a limited number of predictive factors have been established. Future clinical studies have to be accompanied by a translational programme that helps us to identify further predictive markers. From the therapeutic point of view, future focus will be on multi-targeting concepts that include chemotherapy-free regimens. The success of sequential administration of targeted agents in reaching successively prolonged PFS in the sense of turning MBC into a chronic disease, is still under evaluation. In addition, breast cancer stem cell targeting by biologicals opens exciting new therapeutic and preventive perspectives.

\section{References}

1 Hamann PR: Monoclonal antibody-drug conjugates. Expert Opin Ther Patents 2005;15:10871103 .

2 Baselga J, Cameron D, et al.: Objective response rate in a phase II multicenter trial of Pertuzumab (P), a HER' dimerization inhibiting monoclonal antibody, in combination with trastuzumab $(\mathrm{T})$ in patients (pts) with HER2-positive metastatic breast cancer $(\mathrm{MBC})$ which has progressed during treatment with T. J Clin Oncol 2007;25(suppl):abstr 1004.

3 Cameron D, Casey M, et al.: A phase III randomized comparison of lapatinib plus capecitabine versus capecitabine alone in women with advanced breast cancer that has progressed on trastuzumab: updated efficacy and biomarker analyses. Breast Cancer Res Treat 2008;DOI 10,1007/s10549-0079885-0, Epub ahead of print.

4 Wissner A, Mansour TS: The development of HKI272 and related compounds for the treatmen of cancer. Arch Pharm Chem Life Sci 2008;341: 465-477.

5 Burstein HJ, Elias AD, et al.: Phase II study of sunitinib malate, an oral multitargeted tyrosin kinase inhibitor, in patients with metastatic breast cancer previously treated with an anthracycline and a taxane. J Clin Oncol 2008;26:1810-1816.

6 Abraham RT, Eng CH: Mammalian target of rapamycin as a therapeutic target in oncology. Expert Opin Ther Targets 2008;12:209-222.
7 Konecny GE, Meng YG, et al.: Association between HER-2/neu and vascular endothelial growth factor expression predicts clinical outcome in primary breast cancer patients. Clin Cancer Res 2004; 10:1706-1716.

8 Reya T, Clevers H: Wnt signalling in stem cells and cancer. Nature 2005;14:843-849.

9 Slamon DJ, Gomez H, et al.: Randomized phase II study of pazopanib + lapatinib vs. lapatinib alone in patients with HER2+ advanced or metastatic breast cancer. ASCO Meeting Abstracts 2008;26: abstr 1016.

10 Nahta R, Yuan LX, et al.: Lapatinib induces apoptosia in trastuzumab-resitant breast cancer cells:effects on insulin-like growth factor I signalling. Mol Cancer Ther 2007;6:667-674.

11 Arpino G, Wiechmann L, et al.: Crosstalk between the estrogen receptor and the HER tyrosin kinase receptor family: molecular mechanism and clinical implications for endocrine therapy resistance. Endocr Rev 2008;29:217-233.

12 Henriksen KL, Sonne-Hansen K, et al.: Development of new predictive markers for endocrine therapy and resistance in breast cancer. Acta Oncol 2008;47:795-801.

13 Mackey JR, et al.: Trastuzumab and anastrozole in HER2 and hormone receptor co-positive metastatic breast cancer. Breast Cancer Res Treat 2006, oral presentation, abstr 3.

14 O'Shaughnessy J, Blackwell KL, et al.: A randomized study of lapatinib alone or in combination with trastuzumab in heavily pretreated HER2 + metastatic breast cancer progressing on trastuzumab therapy. J Clin Oncol 2008;26(suppl):abstr 1015.
5 Marty M, Cognetti F, et al.: Randomized phase II trail of the efficacy and safety of trastuzumab combined with docetaxel in patients with human epidermal growth factor receptor 2-positive metastatic breast cancer administered as first-line treatment: the M77001 study group. J Clin Oncol 2005;23: 4265-4274.

16 Slamon DJ, Leyland-Jones B, et al.: Use of chemotherapy plus a monoclonal antibody against HER2 for metastatic breast cancer that overexpresses HER2. N Engl J Med 2001;344:783-792.

17 Beachy PA, Karhadkar SS, et al.: Tissue repair and stem cell renewal in carcinogenesis. Nature 2004; 432:324-331, BJC 2004;91:1420-1424.

18 Marx J: Mutant stem cells may seed cancer. Science 2003;301:1308-1310.

19 Kakarala M, Wicha MS: Implications of the cancer stem-cell hypothesis for breast cancer prevention and therapy. J Clin Oncol 2008;26:2813-2820.

$20 \mathrm{Li} \mathrm{X}$, Lewis MT, et al.: Intrinsic resistance of tumorigenic breast cancer cells to chemotherapy. J Natl Cancer Inst 2008;100:672-679.

21 Liu S, Dontu G, et al.: Hedgehog signaling and Bmi1 regulate self-renewal of normal and malignant human mammary stem cells. Cancer Res 2006;66: 6063-6071.

22 Nickoloff BJ. Et al.: Notch signaling as a therapeutic target in cancer: A new approach to the development of cell fate modifying agents. Oncogene 2003;22:6598-6608. 